\title{
Utilização da técnica de ulectomia em Odontopediatria no auxílio da erupção dentária: relato de caso
}

Uso de la técnica de ulectomía en Odontología Pediátrica para ayudar a la erupción dental: informe de un caso Use of the ulectomy technique in Pediatric Dentistry to aid dental eruption: case report

Gabriele Ichara SANCHES ${ }^{1}$

Laura Imbriani BENTO ${ }^{1}$

Nayara Gonçalves EMERENCIANO²

Francyenne Maira Castro GONÇALVES ${ }^{2}$

Marília Andrade Figueiredo de OLIVEIRA ${ }^{1}$

Natália Maria Castorino de OLIVEIRA ${ }^{1}$

Marcelle DANELON ${ }^{3}$

${ }^{1}$ Departamento de Odontologia, Universidade de Ribeirão Preto, UNAERP, 14096-900 Ribeirão Preto - SP, Brasil ${ }^{2}$ Departamento de Odontologia Preventiva e Restauradora, Faculdade de Odontologia, UNESP Univ. Estadual Paulista, 16015-050 Araçatuba - SP, Brasil

${ }^{3}$ Departamento de Odontologia, Universidade de Ribeirão Preto, UNAERP, 14096-900 Ribeirão Preto - SP, Brasil

Departamento de Odontologia Preventiva e Restauradora, Faculdade de Odontologia, UNESP Univ. Estadual Paulista, 16015-050 Araçatuba - SP, Brasil

\begin{abstract}
Resumo
Ulectomia é um procedimento cirúrgico que consiste na remoção dos tecidos que interrompem a erupção dentária. O presente estudo tem como proposição relatar um caso clínico de ulectomia em paciente pediátrico na região do dente 22 . Paciente de 8 anos, do gênero masculino, compareceu a clínica de Odontopediatria da Universidade de Ribeirão Preto (UNAERP) para tratamento odontológico. Ao exame clínico intra-bucal, observou-se aumento volumétrico na região vestibular do dente 22 que ainda não havia erupcionado. A mãe relatou que o filho não apresentou nenhum tipo de trauma naquela região. Após exame radiográfico, observou-se a presença do dente 22 , o qual se encontrava em posição intra-óssea. Assim, foi indicado como forma de tratamento a ulectomia por tratar-se de um procedimento simples e minimamente invasivo. Devido à criança não apresentar bom comportamento diante do exame clínico e radiográfico, optou-se executar inicialmente, sessões de condicionamento para que a seguir, fosse efetuado o procedimento cirúrgico. Após três semanas, foi realizada a cirurgia. Inicialmente foi realizada uma incisão elíptica, onde foi possível a visualização da região incisal do dente 22 . Foi realizado o acompanhamento clínico após 7, 15, 45 e 180 dias, sendo possível observar a erupção do dente 22 . Nos casos de impactações dentárias, há a necessidade de estabelecer a relação com seu fator etiológico para um correto planejamento. Conclui-se que o tratamento realizado proporcionou melhora estética e fonética para o paciente, assim como influenciou no convívio social do mesmo.
\end{abstract}

Descritores: Odontopediatria; Cirurgia Bucal; Erupção Dentária.

\section{Abstract}

Ulectomy is a surgical procedure that consists of removing the tissues that interrupt the tooth eruption. The present study proposes to report a clinical case of ulectomy in a pediatric patient in the tooth 22 region. An 8-year-old male patient attended the Odontopediatrics clinic at the University of Ribeirão Preto (UNAERP) for dental treatment. On intra-oral clinical examination, there was a volumetric increase in the buccal region of tooth 22 that had not yet erupted. The mother reported that the son did not present any type of trauma in that region. After radiographic examination, the presence of tooth 22 was observed, which was in an intraosseous position. Thus, ulectomy was indicated as a form of treatment because it is a simple and minimally invasive procedure. Due to the child not showing good behavior in the face of the clinical and radiographic examination, it was decided to initially perform conditioning sessions so that the surgical procedure could be performed next. After three weeks, surgery was performed. Initially, an elliptical incision was made, where it was possible to visualize the incisal region of tooth 22 . Clinical follow-up was performed after 7, 15, 45 and 180 days, and it was possible to observe the eruption of tooth 22 . In cases of dental impaction, there is the need to establish a relationship with its etiological factor for correct planning. It is concluded that the treatment provided provided aesthetic and phonetic improvement for the patient, as well as influenced the patient's social life.

Descriptors: Pediatric Dentistry; Surgery, Oral; Tooth Eruption.

\section{Resumen}

La ulectomía es un procedimiento quirúrgico que consiste en extraer los tejidos que interrumpen la erupción dental. El presente estudio propone informar un caso clínico de ulectomía en un paciente pediátrico en la región del diente 22 . Un paciente masculino de 8 años asistió a la clínica de odontología de la Universidad de Ribeirão Preto (UNAERP) para recibir tratamiento dental. En el examen clínico intraoral, hubo un aumento volumétrico en la región bucal del diente 22 que aún no había erupcionado. La madre informó que el hijo no presentaba ningún tipo de trauma en esa región. Después del examen radiográfico, se observó la presencia del diente 22 , que estaba en posición intraósea. Por lo tanto, la ulectomía se indicó como una forma de tratamiento porque es un procedimiento simple y mínimamente invasivo. Debido a que el niño no mostró un buen comportamiento frente al examen clínico y radiográfico, se decidió realizar inicialmente sesiones de acondicionamiento para que el procedimiento quirúrgico pudiera realizarse a continuación. Después de tres semanas, se realizó una cirugía. Inicialmente, se realizó una incisión elíptica, donde fue posible visualizar la región incisal del diente 22 . El seguimiento clínico se realizó después de 7, 15, 45 y 180 días, y fue posible observar la erupción del diente 22. En casos de impactación dental, hay La necesidad de establecer una relación con su factor etiológico para una correcta planificación. Se concluye que el tratamiento proporcionado proporcionó una mejora estética y fonética para el paciente, así como también influyó en la vida social del paciente.

Descriptores: Odontología Pediátrica; Cirugíal Bucal; Erupción Dental.

INTRODUÇÃO

O aparecimento da coroa dentária na cavidade oral é um processo fisiológico que consiste na migração do germe de sua posição intraóssea, tanto na maxila quanto mandíbula, até a sua posição funcional ${ }^{1}$. Esse fenômeno é denominado erupção dentária, representando parte do desenvolvimento e crescimento infantil. A cronologia de erupção é definida a 
partir de fatores genéticos, sistêmicos e ambientais ${ }^{2-8}$, apresentando diferenças em seu desenvolvimento causado por fatores como o nível socioeconômico, gênero, área geográfica, nascimento prematuro, etnia e estado nutricional infantil ${ }^{9}$. Hipotireoidismo, fibrose gengival, presença de dentes supranumerários, desnutrição, traumatismos, tumores, cistos dentígeros, ausência de dentes, são fatores que alteram esta cronologia. Nos últimos anos, este assunto tem sido muito estudado, mas até hoje só há teorias sobre 0 assunto. Contudo, acredita-se que está diretamente associado com o crescimento da face, dos ossos, ação dos músculos e forças oclusais ${ }^{10,11}$.

A falha na erupção dentária pode ser primária, quando há mal funcionamento do mecanismo erupcionário, ou secundário, quando o dente encontra-se em processo de erupção, mas se torna anquilosado e com isso perde a capacidade de manter o processo eruptivo. Os dentes decíduos têm histórico raro de impactação, encontrados mais comumente anquilosados. Porém, os dentes permanentes que mais preocupam quando não presentes no tempo cronológico, são os incisivos centrais superiores, pois esse influencia diretamente na estética facial da criança e afeta também a fala, mastigação e o psicológico. Alguns estudos mostram que a retenção do dente está ligada à fibrose gengival que é mais comum em incisivos e caninos ${ }^{12}$.

Quando ocorre retardo da erupção dentária, o cirurgião-dentista pode realizar um procedimento cirúrgico denominado de ulectomia, que consiste na exérese dos tecidos que estão interrompendo 0 processo de erupção, logo, tem como principal função permitir que o dente ocupe sua posição correta no arco dentário ${ }^{13}$. É realizado em dentes permanentes quando surgem na cavidade bucal, mas por algum motivo não tem forças para erupcionar, demorando a tomar sua posição na arcada, sendo assim, este procedimento pode evitar a pericoronarite. Cistos ou hematomas de erupção, embora não tenham como primeira escolha a ulectomia, ela pode ser indicada quando houver inflamação ${ }^{14}$. ou quando clinicamente se apresentar como uma área com aumento volumétrico e de coloração pálida ou azulada, além de marcas contornadas, denotando a presença do dente não irrompido ${ }^{15,16}$. Entretanto, uma correta indicação de ulectomia só pode ser considerada eficaz, após a realização de exame clínico e radiográfico.

A técnica de ulectomia é um procedimento de fácil execução, que envolve: anestesia local da mucosa, incisão, exposição da coroa dentária, remoção da mucosa e hemostasia. Promove a remoção de uma faixa de mucosa, em forma elíptica, expondo assim toda a borda incisal do dente afetado, podendo este irromper espontaneamente, ou necessitar da ajuda de aparelhos ortodônticos com força extrusiva $^{17}$. O dente precisa irromper e tomar seu lugar na cavidade oral no tempo certo, o atraso ou a retenção pode causar problemas tanto na dentadura mista quanto decídua, tendo os problemas de má oclusão como exemplo. Em raríssimos casos, devido a sua complexidade, se faz necessário o acompanhamento com um ortodontista, pois poderá ocorrer a não erupção. Além de ser uma cirurgia de baixa complexidade, as crianças acompanhadas após alguns anos de cirurgia, só nos mostra o quanto a ulectomia pode ajudar o indivíduo ${ }^{18}$.

\section{CASO CLÍNICO}

Paciente do sexo masculino, 8 anos de idade, compareceu a clínica de Odontopediatria da Universidade de Ribeirão Preto (UNAERP), para tratamento odontológico. Durante anamnese e exame clínico, constatou a ausência do elemento 22 com aumento volumétrico e mãe relatou não ter sofrido nenhum tipo de alteração sistêmica, assim como, trauma ou perda precoce do dente decíduo. A Figura 1 mostra o aspecto clínico inicial. Mucosa na região do dente 22 com aspecto rosa pálido e tecido gengival fibroso. Ao exame radiográfico foi observado coroa totalmente formada, raíz com rizogênese incompleta e uma fina camada de tecido ósseo sobre 0 dente 22 (Figura 2).

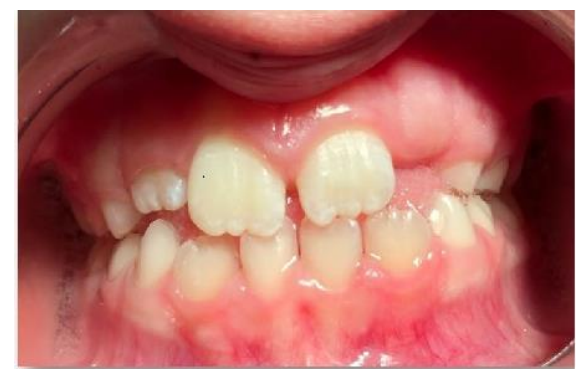

Figura 1: Exame clínico inicial.

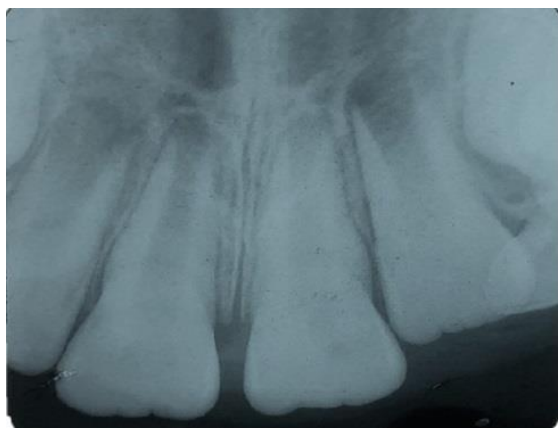

Figura 2: Exame radiográfico inicial. 
Foi utilizada anestesia tópica com gel de Benzocaína (Benzocaíne ${ }^{\circledR}$ Interstate Drug Exchange, IL, EUA) (Figura 3), e em seguida a anestesia terminal infiltrativa usando anestésico Citocaína 3\% (Cristália ${ }^{\circledR}$, cloridrato de prilocaína com felipressina), nas áreas de fundo de fórnix da região de mucosa do dente 22 anestesiando o nervo alveolar superior anterior (Figura 4).

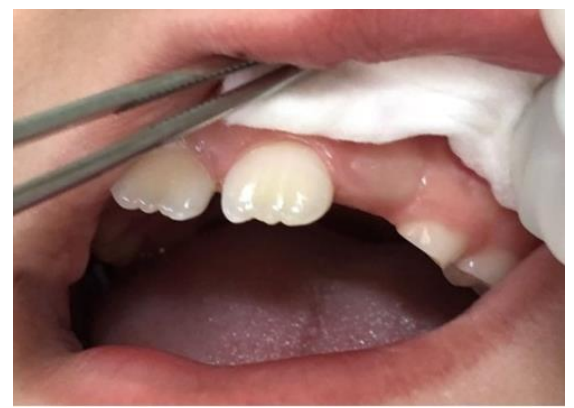

Figura 3: Anestesia tópica da área com Benzocaína (Benzocaíne ${ }^{\circledR}$, por 3 minutos).

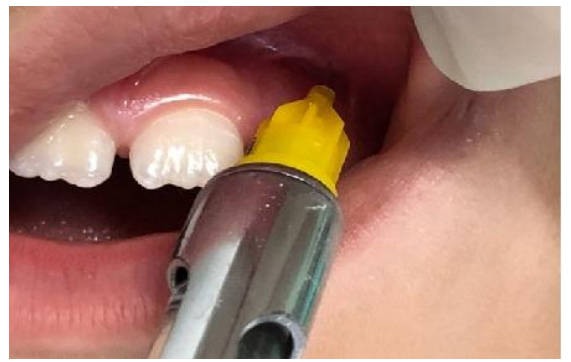

Figura 4: Anestesia terminal infiltrativa (Citocaína 3\%).

Com o auxílio de lâmina de bisturi no 15 (Lunamed, São Paulo, Brasil) realizou-se uma incisão de forma elíptica ao redor da mucosa a ser removida (Figura 5). Após diérese dos tecidos com pinça hemostática (Figura 6) foi possível observar a incisal do dente 22 (Figura 7). A região foi irrigada com soro fisiológico e pressionada com gaze estéril, para promover hemostasia. No pós-operatório de 7, 15,45 e 180 dias foi possível visualizar a erupção do elemento dentário (Figuras 8 e 9).

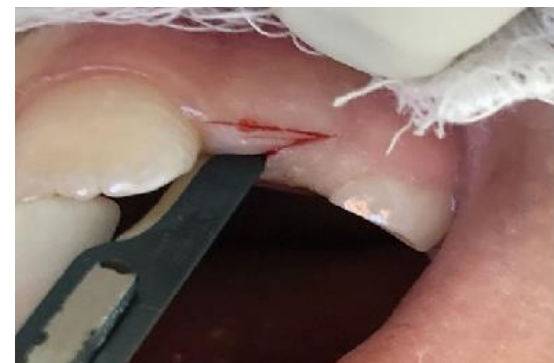

Figura 5: Incisão elíptica com lâmina de bisturi no 15

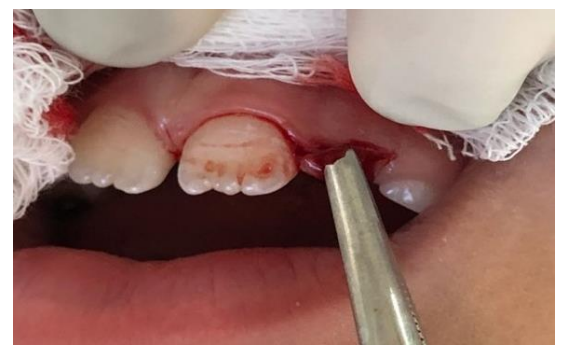

Figura 6: Remoção do tecido gengival remanescente com pinça hemostática.

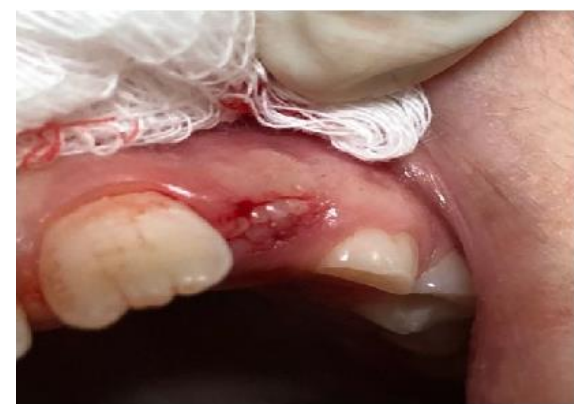

Figura 7: Pós-cirúrgico imediato.

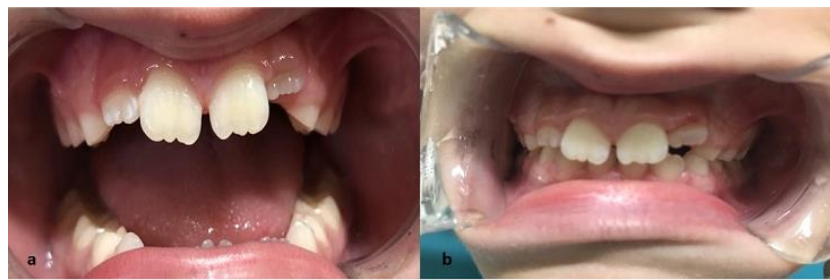

Figura 8: Aspecto clínico após 7 (a) e 15 (b) dias da ulectomia.

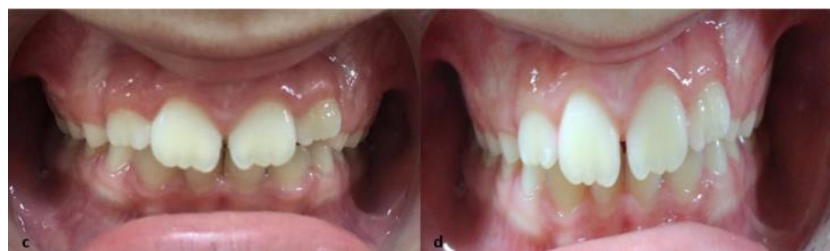

Figura 9: Aspecto clínico após 45 (c) e 180 (d) dias da ulectomia.

DISCUSSÃO

A erupção dentária é um processo fisiológico, que segundo Enwonwu ${ }^{2}$, é definido a partir de fatores genéticos, sistêmicos e hereditário, mas até sua total erupção podem ocorrer diversos problemas, atrapalhando sua perfeita erupção causando problemas de máoclusão, assim é necessário exames para verificação de uma possível agenesia ou apenas retardo dentário.

Para Cavalcante e Paiva ${ }^{17}$, o exame clínico e radiográfico, auxiliam no diagnóstico, das possíveis causas relacionadas a ausência da erupção, como odontomas, dentes supranumerários e/ou impactados. Assim, indicando o procedimento corretamente, ou até mesmo contraindicando, em casos de uma grande camada óssea recobrindo a coroa dentária. No presente caso clínico o exame radiográfico foi de extrema impotância para se constar a presença ou ausência dos fatores acima mencionados.

Guedes-Pinto $^{15}$ orienta que, ao expor a coroa dentária, que pode ainda estar recoberta por tecido ósseo alveolar, deve-se efetuar cuidadosamente a osteotomia, caso seja necessário. Neste relato de caso, após a incisão gengival, não foi observado nenhuma camada de tecido ósseo, assim, visualizando imediatamente a incisal do dente, não sendo necessário tal procedimento. Para Martinez et al. $^{19}$, o exame radiográfico do estágio de rizogênese é importante, devendo corresponder 
ao estágio 8 de Nolla (2/3 da raíz formada) para a indicação desta técnica que é o caso do relato em questão. Já Issao, Guedes-Pinto ${ }^{20}$ (1994) relataram que a partir do $7^{\circ}$ estágio de Nolla (1/3 de raíz formada) o dente já apresenta força eruptiva, sendo, portanto, a ulectomia indicada. Cavalcante, Paiva ${ }^{17}$ e Issao e GuedesPinto $^{20}$ recomendam realizar a ulectomia logo após a indicação, evitando possíveis movimentações indesejáveis, como fechamento de espaços, inclinação de dentes adjacentes, extrusão do antagonista, implicando diretamente a um possível tratamento ortodôntico. Além de poder causar curvamento radicular.

Durante o procedimento cirúrgico, indicase incisão de forma elíptica permitindo a exposição da incisal ou face oclusal do dente, podendo ser realizada com lâmina de bisturi, laser ou bisturi elétrico, removendo-se qualquer interferência ${ }^{21}$. Quando a cirurgia envolve apenas tecido gengival, o pós-operatório é favorável, não sendo relatado nenhum tipo de sintomatologia significativa. No presente caso, o paciente não relatou incômodo após 0 procedimento cirúrgico. Além de restabelecer a função do elemento dentário na arcada, um correto diagnóstico pode influenciar no convívio social da criança, uma vez que a mesma terá maior estímulo à manutenção do convívio social. Dessa forma, um dente que tem a sua cronologia de erupção alterada pode gerar uma repercussão negativa no desenvolvimento emocional e na vida daquele indivíduo. No presente caso, foi observado segundo relatos dos acompanhantes que a criança melhorou sua relação com amigos da escola.

CONCLUSÃO

A ulectomia é um procedimento cirúrgico minimante invasivo que consiste na remoção de tecidos que estão interferindo a erupção dentária, expondo a coroa do dente resultando em uma erupção espontânea. Os exames clínico e radiográfico são indispensáveis para realização desta técnica.

No caso relatado, a indicação de ulectomia foi eficaz, visto que no momento da cirurgia, a incisal do dente já havia sido exposta.

Conclui-se que o tratamento realizado proporcionou melhora estética e fonética para o paciente, assim como influenciou no convívio social do mesmo.

\section{REFERÊNCIAS}

1. Kolokitha OE, Papadopoulou AK. Impaction and apical root angulation of the maxillary central incisors due to supernumerary teeth: Combined surgical and orthodontic treatment. Am J Orthod Dentofacial Orthop. 2008;134:153-60.
2. Enwonwu CO. Influence of socio-economic conditions on dental development in Nigerian children. Arch Oral Biol. 1973;18:95-107.

3. Singh N, Sharma S, Sikri V, Singh P. To study the average age of eruption of primary dentition in Amritsar an surrounding area. J Indian Dental Assoc. 2000;71:26.

4. Haddad AE, Correa MS. The relationship between the number of erupted primary teeth and the child's height and weight: a crosssectional study. J Clin Pediatr Dent. 2005;29:3357-362.

5. Holman DJ, Yamaguchi K. Longitudinal analysis of deciduous tooth emergence: IV Covariate effects in Japanese children. Am J Phys Anthropol. 2005;126:352-58.

6. Bastos JL, Peres MA, Peres KG, Barros AJ. Infant grown, development and tooth emergence patterns: a longitudinal study from birth to 6 years of age. Arch Oral Biol. 2007;52:598-606.

7. Folayan M, Owotabe F, Adejuyigbe E, Sen S, Lawal B, Ndukwe K. The timing of the primary dentition in Nigerian children. Am $J$ Phys Anthropol. 2007; 134:443-48.

8. Oziegbe EO, Adenoyasofowora C, Folayan MO, Esan TA, Owotabe FJ. Relationship between sociodemographic and anthropometrics variables and number of erupted primary teeth in suburban Nigerian children. Matern Child Nutr. 2009;5:86-92.

9. Duarte $M E Q$, Andrade MA, Faria PC, Marques LS, Jorge MLR. Fatores associados à cronologia de erupção de dentes decíduos Revisão de Literatura: Erupção de dentes decíduos e fatores associados. Rev Univ Vale do Rio Verde. 2011;9:139-51.

10. Huber K, Suri L, Parul T. Eruption Disturbances of the maxillary incisors: a literature review. J Clin Pediatr Dent. 2008;32:221-30.

11. Candeiro GTM, Correia FC, Candeiro SALM. Ulectomia como opção cirúrgica no retardo da erupção dentária: relato de caso. Rev Odontol Araçatuba. 2009;30:45-9.

12. Giglio FPM, Gurgel JA. Abordagem cirúrgicoortodôntica de dentes não irrompidos. Ortodontia. 2010;43:169-75.

13. Arnaud RR, Santos MGC, Valença AMG, Forte FDS, Lima KJR, Beltão RTS. Ulotomia: coadjuvante do tratamento da má oclusão. RFO. 2014;19:234-38.

14. Pinkham JR, Casamassimo PS, Fields HW, McTigue DJ, Nowak A. Odontopediatria da Infância à Adolescência. São Paulo: Artes Médicas; 1996.

15. Guedes-Pinto AC. Odontopediatria. São Paulo: Santos; 2010.

16. Candeiro GTM, Correia FC, Candeiro SALM. Ulectomia como opção cirúrgica no retardo da erupção dentária: relato de caso. Rev Odontol Araçatuba. 2009;30:45-49. 
17. Cavalcante AL, Paiva LCA. Utilização da ulectomia na clínica infantil: relato de caso. UEPG Ci Biol Saúde. 2006;12:39-42.

18. Biagi E, Butti AC. Premature loss of maxillary primary incisor and delayed eruption of its successor: Report of a case. Eur J Paediatr Dent. 2011;12:194-97.

19. Martinez JA, Mota LFG, Miranda IMAD, Guedes-Pinto AC. Cirurgia em odontopediatria. São Paulo: Artes Médicas; 1998.

20. Issao M, Guedes-Pinto AC. Manual de Odontopediatria. São Paulo: Pancast; 1994.

21. Poricelli E, Ponzoni D. Cirurgia bucal pediátrica. In: Toledo OA. Odontopediatria: fundamentos para a prática clínica. São Paulo: Premier; 2005.

\section{CONFLITO DE INTERESSES}

Os autores declaram não haver conflitos de interesse

\section{AUTOR PARA CORRESPONDÊNCIA}

\section{Marcele Danelon}

Departamento de Odontologia,

Universidade de Ribeirão Preto - UNAERP,

Av Costábile Romano 2.201

14096-900 Ribeirão Preto - SP, Brasil

Tel. +551636036717

E-mail: marcelledanelon@hotmail.com 